\title{
Enjeux, difficultés et modalités de l'expression sur le travail : point de vue de la clinique médicale du travail
}

Concerns in occupational medicine about stakes, difficulties and conditions of dispute about work

Retos, dificultades y modalidades de la expresión sobre el trabajo : punto de vista de la clínica médica del trabajo

\section{Philippe Davezies}

\section{(2) OpenEdition}

Édition électronique

URL : http://journals.openedition.org/pistes/2566

DOI : $10.4000 /$ pistes. 2566

ISSN : 1481-9384

Éditeur

Les Amis de PISTES

Référence électronique

Philippe Davezies, «Enjeux, difficultés et modalités de l'expression sur le travail : point de vue de la clinique médicale du travail », Perspectives interdisciplinaires sur le travail et la santé [En ligne], 14-2 | 2012, mis en ligne le 01 novembre 2012, consulté le 02 mai 2019. URL : http:// journals.openedition.org/pistes/2566; DOI : 10.4000/pistes.2566

Ce document a été généré automatiquement le 2 mai 2019.

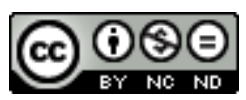

Pistes est mis à disposition selon les termes de la licence Creative Commons Attribution - Pas d'Utilisation Commerciale - Pas de Modification 4.0 International. 


\title{
Enjeux, difficultés et modalités de l'expression sur le travail : point de vue de la clinique médicale du travail
}

\author{
Concerns in occupational medicine about stakes, difficulties and conditions of \\ dispute about work \\ Retos, dificultades y modalidades de la expresión sobre el trabajo : punto de vista \\ de la clínica médica del trabajo
}

Philippe Davezies

\section{NOTE DE L'ÉDITEUR}

Cet article est commenté par Pascal Vitte, le commentaire peut être consulté à l'adresse : http://pistes.revues.org/3457 On retrouve aussi la réponse de Philippe Davezies au commentaire de Pascal Vitte :

http://pistes.revues.org/3462

Face aux expressions de souffrance psychique qui lui sont adressées, le professionnel de santé au travail alerte la direction de l'entreprise et les instances représentatives du personnel. Malheureusement, dans de nombreux cas, cette alerte n'est pas suivie de mesures susceptibles d'améliorer la situation. Une des raisons de ce qui apparaît comme un échec tient au fait que les destinataires de l'alerte sont déjà plus ou moins confusément informés de l'existence d'un problème, qu'ils ne savent pas comment l'aborder, et que l'alerte ne leur ouvre pas de nouvelles perspectives de compréhension et d'action. Pour espérer ouvrir des perspectives de transformation, il est nécessaire de reprendre les choses du point de vue du travail, de ses tensions, de ses impasses, mais 
aussi des perspectives que révèle son analyse. Conformément au titre d'un ouvrage classique, il faut « Comprendre le travail pour le transformer » (Guérin, 1997).

\section{L'activité et ses dilemmes}

2 Dans cette direction, l'analyse se heurte immédiatement à un obstacle : la représentation du travail qui circule dans l'entreprise, celle qui est la plus facile à exprimer et qui est toujours mise en avant, c'est celle qui est donnée par les consignes. Or, la réalité du travail est beaucoup plus large.

\subsection{Au-delà de la prescription, la dynamique de l'activité}

3 C'est la leçon fondamentale de l'ergonomie : le travail n'est jamais la simple réalisation de la tâche telle qu'elle est formulée par la prescription. Travailler impose toujours de prendre en charge des particularités de la situation que la hiérarchie n'est pas en état de percevoir.

4 L'analyse ergonomique met l'accent sur les éléments de variabilité qui contraignent le travailleur à prendre des distances avec les modes opératoires prescrits pour parvenir, malgré tout, aux résultats fixés par la hiérarchie. Et, effectivement, dans la phase de prise en main du travail, l'enjeu pour le travailleur consiste à montrer qu'il maîtrise la situation et qu'il est capable de répondre aux attentes de la hiérarchie. Cependant, une fois cette étape franchie, l'attention, libérée par l'acquisition et l'incorporation des savoir-faire, se reporte sur d'autres enjeux de l'activité. Il apparait au travailleur que sa façon de travailler a, sur la qualité de la production, sur les relations avec les collègues, sur l'état des installations ou de l'environnement, des répercussions vis-à-vis desquelles sa hiérarchie ne manifeste pas d'attente particulière parce que bien souvent elle n'en perçoit pas l'importance. Il y répond alors en mobilisant son expérience, sa sensibilité, ses valeurs (Davezies, 2006).

5 Le travail engage ainsi le salarié dans une dynamique d'élargissement de la zone vis-à-vis de laquelle il prétend assumer une responsabilité. Ce développement du pouvoir d'agir, qui porte le sujet au-delà de ses intérêts personnels directs, humanise le travail en lui donnant une forme dans laquelle le salarié peut se reconnaître.

6 Ce mouvement n'a pas échappé aux recherches dans le domaine du management. La littérature américaine a une expression pour en rendre compte: Organizational Citizenship Behavior - comportement de citoyenneté organisationnelle. Cette notion désigne la prise en considération des intérêts collectifs et le développement par le travailleur d'une activité propre, au-delà de ce qui est prescrit. Smith souligne le fait qu'il ne s'agit pas d'un phénomène marginal :

«Every factory, office, bureau depends daily on a myriad of acts of cooperation, helpfulness, suggestion, gesture of goodwill, altruism, and other instances of what we might call citizenship behavior.» (Smith, 1983).

7 Le mouvement qui porte les participants à aller au-delà de ce qui est prescrit est une condition essentielle de la survie et du développement de l'organisation (Organ, 1990). 


\subsection{Intensification et dégradation de la qualité}

8 La situation se complique pourtant sérieusement à partir du moment où l'organisation se retourne contre ce mouvement pourtant essentiel à son fonctionnement. C'est ce qui s'est produit du fait de la pression à l'accélération et à la standardisation qui a caractérisé le mouvement d'intensification du travail des dernières décennies (Davezies, 2005 ; Clot, 2010).

9 À tous niveaux hiérarchiques, les travailleurs se retrouvent en situation de devoir satisfaire les objectifs très tendus qui leur sont imposés tout en assurant comme ils le peuvent le fonctionnement global de l'organisation et en s'efforçant de lui donner figure humaine. Pour la plupart, cela se traduit par des situations où il n'est pas possible de prendre en charge tout ce qu'il y aurait à faire pour que les choses se passent bien. Chacun doit trier entre les objectifs qu'il va endosser et ceux qu'il lui faudra bien laisser couler. Or, il n'y a pas de dispositifs sociaux pour discuter des dilemmes éthiques de l'activité et des choix qui se présentent nécessairement. Sous la pression de l'intensification, les espaces de discussion ont plutôt reculé. Chacun trie donc à sa façon, en fonction de son expérience, de sa sensibilité, de son histoire. Il ne s'agit pas là d'individualisme, mais des effets d'une organisation du travail qui impose des arbitrages serrés qu'elle renvoie aux choix individuels. Cette individualisation du rapport au travail fragilise considérablement la capacité d'expression de l'expérience du travail face aux normes managériales. Une part importante des questions vives du travail n'est pas mise en débat, mais se manifeste sous la forme de conflits extrêmement individualisés (Béroud, 2008).

\subsection{La nécessité d'espaces de discussion sur le travail}

Dans ce contexte, de nombreuses voix plaident en faveur du développement, au sein des entreprises, d'espaces de discussion qui ne se limitent pas à une communication descendante, mais permettent l'expression et la prise en charge collective des dilemmes de l'activité.

11 Dans le domaine de l'épidémiologie, le modèle de la justice organisationnelle est ainsi apparu comme un indicateur de stress professionnel complémentaire des modèles de Karasek (1990) et de Siegrist (1998). Ce nouveau modèle comporte, dans sa version la plus utilisée par les épidémiologistes, une dimension de justice procédurale qui vise les modalités de la prise de décision dans l'entreprise et les possibilités d'expression et d'intervention des salariés (Elovainio, 2002). Les recherches menées sur la base de ce modèle montrent que la situation dans laquelle les salariés se voient imposer les décisions sans pouvoir les discuter constitue un facteur d'augmentation des troubles du sommeil (Elovainio, 2009), des syndromes métaboliques (Gimeno, 2010), des marqueurs de l'inflammation (Elovainio 2010), du tabagisme (Kouvonen, 2007), des excès de consommation alcoolique (Kouvonen, 2008), de l'absentéisme pour maladie (Elovainio, 2002), des pathologies cardiovasculaires (Elovainio, 2006) et des dépressions (Ylipaavalniemi, 2005).

12 Le même souci de promouvoir des formes de démocratie dans l'entreprise est exprimé du côté du management. Le modèle de la justice organisationnelle avait d'ailleurs émergé dans le champ des ressources humaines, au nom d'un souci d'amélioration des 
performances de l'entreprise, avant d'être repris par l'épidémiologie. Ainsi, la Chambre de commerce et d'industrie de Paris fait l'éloge du management participatif, donné comme une source d'économie et de renforcement de la compétitivité (Chambre de commerce et d'industrie de Paris, 2007). La justice procédurale favoriserait le développement de la citoyenneté organisationnelle (Moorman, 1991; 1993). La notion d 'empowerment qui a cours dans la littérature managériale véhicule des conceptions très voisines (Gilbert, 2010).

Conformément à cette logique, la norme ISO 26000 sur la responsabilité sociétale des entreprises met l'accent sur le développement du dialogue social :

«Un dialogue social effectif fournit un mécanisme permettant d'élaborer une politique ou de trouver des solutions prenant en compte les priorités et les besoins des employeurs et des travailleurs, ceci aboutissant à des résultats qui sont valables et durables, tant pour l'organisation que pour la société. Le dialogue social peut contribuer à instituer la participation et des principes démocratiques sur les lieux de travail, à assurer une meilleure compréhension entre l'organisation et ceux qui réalisent le travail et à établir de saines relations entre le monde du travail et le management, réduisant ainsi le risque de conflits sociaux coûteux. » (NF ISO 26000, 2010)

Le rapport Lachmann au premier ministre de la France affirme aussi la nécessité de restaurer des espaces de discussion et d'autonomie dans le travail :

«La performance et l'innovation font appel à l'intelligence collective des salariés : les occasions de leur redonner de l'initiative doivent donc être favorisées. Tout salarié veut être efficace et utile, c'est ce qui donne du sens et de la fierté à son travail. Il est souvent le mieux placé pour identifier les dysfonctionnements et proposer des pistes d'amélioration de l'efficacité. [...] Les espaces de régulation et de discussion sont donc indispensables, à plusieurs titres : pour que les salariés s'approprient leurs pratiques professionnelles sur le plan technique et éthique, pour donner sa place à la performance collective dans le travail, et enfin pour prévenir les conflits au travail par des espaces de partage et de dialogue sur les difficultés rencontrées. » (Lachman, 2010

L'idée selon laquelle la prise en compte de l'expérience des travailleurs constituerait un enjeu à la fois pour la santé des salariés et pour la performance de l'entreprise est donc couramment avancée de divers côtés. Or, le droit d'expression est inscrit dans le Code du travail français (articles L2281-1 et suivants).

Des raisons de nature très diverses ont contribué à ce que ce pan du droit reste lettre morte. Nous nous en tiendrons à la plus élémentaire, celle qui concerne la difficulté des individus à parler de leur travail, dans la mesure où elle constitue un défi central pour les professionnels de santé, pour les représentants du personnel et pour la hiérarchie.

\section{Un défi : l'obscurité de l'activité}

Nous touchons ici la question centrale à partir de laquelle se construisent les approches cliniques du travail (ergonomie, ergologie, psychodynamique, clinique de l'activité, clinique médicale du travail) : il existe une distance parfois très importante entre ce que fait le travailleur et ce qu'il est en mesure d'en dire. Cette difficulté à dire le travail ne relève pas d'une déficience intellectuelle. La capacité à extraire de l'expérience des éléments susceptibles de constituer une ressource partagée pour l'avenir est une tâche compliquée, aussi bien pour le psychiatre ou le chercheur que pour l'ouvrier. Sennet (2010) évoque ainsi l'exaspération des réformateurs du système de santé publique 
britannique devant l'incapacité des médecins et infirmières à rendre compte des ressorts de leurs activités. Cette obscurité de l'action aux yeux de celui qui l'exécute trouve son origine dans la physiologie du système nerveux. Une série de résultats expérimentaux permettent d'en prendre la mesure.

\subsection{Les programmes moteurs acquis au cours des apprentissages ne sont pas directement accessibles à l'expression verbale}

18 C'est un fait bien connu : le sujet n'a qu'une conscience limitée de la façon dont il réalise les tâches dans lesquelles il est engagé. Celles-ci mobilisent la mémoire procédurale qui fait partie des mémoires implicites, c'est-à-dire des mémoires qui ne sont pas accessibles à la conscience. Chacun sait nouer ses lacets, mais c'est une toute autre affaire que d'expliquer comment, lorsque nous tenons une extrémité du lacet dans la main droite et l'autre dans la main gauche, nous faisons le nœud. De même, le potier sera bien embêté si on lui demande d'expliquer comment, sur son tour, il fait sortir un pot de la masse d'argile. Plutôt que de tenter d'expliquer, il préférera montrer (Malafouris, 2008). Dans le domaine sportif, chacun sait que le mouvement s'enraye à partir du moment où il doit être piloté par le raisonnement. Pour se déployer de façon efficace, le geste implique une conscience du but, mais la réalisation doit lâcher la bride à la mobilisation corporelle.

\subsection{Le cerveau efface une grande partie des informations sensorielles émanant du mouvement en cours}

19 Non seulement nous mobilisons des programmes moteurs dont nous n'avons pas une claire conscience, mais le cerveau tend à ignorer une grande partie des informations sensorielles qui pourraient nous renseigner sur le mouvement une fois qu'il est lancé. Le déclenchement d'un mouvement a pour corollaire la production d'un modèle interne, appelé copie d'efférence, qui anticipe le mouvement à venir et ses conséquences prévisibles en matière de retour sensoriel. Dans la formulation proposée par Von Holtz (1980), tout se passe comme si les informations sensorielles anticipées étaient marquées d'un signe plus et les informations sensorielles traduisant ce qui se produit réellement d'un signe moins ; le cerveau ne traiterait que leur résultante. Si tout se déroule comme prévu par le programme sensorimoteur, la comparaison des deux types d'informations ne laisse pas de résidu, les informations sensorielles sur le mouvement sont supprimées. Au contraire, une différence est le signe d'une influence extérieure au mouvement qui impose un ajustement de l'action. Seul ce résidu fait l'objet d'un traitement. Le cerveau dispose donc d'un mécanisme qui efface en grande partie les retours sensoriels dès lors qu'ils ne sont que les conséquences du mouvement engagé. C'est ce qui explique que l'on n'obtienne pas le même résultat en se chatouillant soi-même et si l'on est chatouillé par son voisin. Ce dispositif permet de faire la part entre ce qui relève du mouvement provoqué par la personne et ce qui relève d'une influence de l'environnement. Sa perturbation est évoquée comme responsable de l'attribution erronée de ses actes à des forces extérieures par le schizophrène (Shergill, 2005 ; Frith, 2005; 2010). Cette régulation, dite en feedforward, qui ne vise que ce qui ne se passe pas comme anticipé, permet un ajustement beaucoup plus rapide qu'une simple régulation en feed-back.

Nous aboutissons donc à un constat troublant : l'efficacité de la régulation du geste et la capacité à se percevoir comme l'agent de l'action supposent une certaine opacité de celle- 
ci pour l'agent lui-même. Au bout du compte, le sujet perçoit son propre mouvement parce qu'il se voit agir beaucoup plus qu'il ne se sent agir (Jeannerod, 2009).

\subsection{Les ajustements de la gestuelle n'impliquent pas la conscience}

21 Si le cerveau valorise les informations sensorielles qui alertent sur ce qui ne se déroule pas comme anticipé, cela ne signifie pas pour autant que leur traitement implique la conscience. Fourneret et Jeannerod (1998) ont montré que, pour peu qu'il ne soit pas majeur, l'ajustement est réalisé sans que le sujet n'ait conscience d'avoir modifié son geste au cours de sa réalisation. Citer ici toutes les observations qui démontrent cette capacité à réguler le geste indépendamment de la conscience dépasserait le cadre du présent article. Nous nous en tiendrons à un des faits les plus frappants : l'existence d'une vision aveugle, c'est-à-dire d'une capacité des sujets qui présentent une cécité liée à une lésion du cortex visuel à pointer du doigt correctement un signal que pourtant ils n'ont pas conscience de voir (Weiskrantz, 1974; Danckert, 2005). Il apparait ainsi que l'information sensorielle n'est pas traitée de la même façon pour la perception consciente et pour l'action. Ces travaux expérimentaux confirment ce que nous avaient enseigné les observations sur le travail : même sur des activités gestuelles en principe très réglées, il y a une différence parfois importante entre le geste tel que les gens se le représentent et celui qu'ils exécutent.

\subsection{La préparation de l'action est lancée avant la décision consciente d'agir}

Le résultat certainement le plus contraire au sens commun concerne les relations entre la décision et l'action. Benjamin Libet $(1983 ; 1999)$ a demandé à un sujet d'appuyer sur un bouton quand il le voulait, en notant le moment de sa prise de décision. L'enregistrement électro-encéphalographique a révélé l'apparition d'un potentiel de préparation enregistrable dans l'aire prémotrice avant que le sujet n'ait pris la décision d'agir. L'observation a été confirmée par d'autres auteurs. Haggard a montré que, dans des situations où le sujet devait choisir de répondre avec la main droite ou la main gauche, un potentiel de préparation latéralisé permettait de prédire le mouvement avant que le sujet n'ait opéré son choix et pris sa décision (Haggard, 1999; 2008). L'expérience a été reproduite avec des malades auxquels avaient été implantées des électrodes afin de localiser l'origine de crises épileptiques. L'enregistrement d'une activité dans l'aire motrice supplémentaire, une seconde et demie avant la décision, permettait de prévoir celle-ci et de prédire le moment de son apparition (Fried, 2011). L'utilisation de la résonance magnétique fonctionnelle a permis de remonter plus en amont dans les phénomènes de préparation de l'action. Avant les aires prémotrices, une activation était observée dans le cortex préfrontal et dans le cortex pariétal. Là encore l'observation de l'activité cérébrale permettait de prévoir le côté choisi pour le mouvement avant que le sujet n'ait pris sa décision (Soon, 2008). De ces travaux, il ressort que le processus qui conduit à l'action débute avec une intention d'agir inconsciente (Sirigu, 2004; Daprati, 2010) ; celle-ci se manifeste par une activation du cortex préfrontal, qui constitue le plus haut niveau d'intégration de l'expérience, et du cortex pariétal dont le fonctionnement est nécessaire à la prise de conscience de l'intention motrice, à la production du modèle interne du mouvement et à la conscience du corps. Cette intention inconsciente produit 
une activation du système moteur dont l'indice est l'apparition d'un potentiel de préparation environ une seconde à une seconde et demie avant la réponse motrice. $\mathrm{Ce}$ potentiel de préparation se latéralise en fonction du côté du mouvement, 800 millisecondes avant la contraction musculaire. L'intention consciente n'émerge que 250 millisecondes avant le déclenchement du geste (Desmurget, 2009). Ces 250 millisecondes sont le temps nécessaire à la planification des commandes motrices correspondant au mouvement désiré. En somme, l'intention consciente accompagne le déclenchement de l'action, elle ne la précède pas. En cas d'urgence, ce dispositif est court-circuité par un système sous-cortical plus rapide dans lequel l'amygdale joue un rôle déterminant (Jeannerod, 2009). Dans tous les cas, la décision d'agir n'est pas la cause de l'action.

\subsection{Les éléments de la situation qui appellent le déclenchement de l'action ne sont qu'en partie conscients}

Le fait d'avoir présenté à un sujet, au cours d'une tâche immédiatement antérieure, des stimuli (des mots ou des figures) évoquant des enjeux sociaux est capable de déclencher chez lui, dans la situation suivante, un comportement orienté par ces enjeux, de renforcer la mobilisation en ce sens, d'accroître la capacité à persister malgré les obstacles, de favoriser la reprise après interruption même en présence d'alternatives plus attirantes, sans que le sujet n'ait conscience de l'influence des signaux qu'il a perçus sur son comportement (Bargh, 2001; Custers, 2008). Des résultats du même type sont observés dans des situations où le stimulus qui va orienter l'action fait l'objet d'une présentation subliminale et n'est donc même pas consciemment perçu (Strahan, 2002 ; Aarts, 2005 ; Custers, 2010). Le sujet a conscience du but poursuivi, même si cette conscience est en retard par rapport à l'actualisation des programmes moteurs; il se voit agir, il peut constater le résultat de son action; en revanche, il n'a qu'une conscience très partielle de ce qui le conduit à sélectionner et à maintenir le but sur lequel il est engagé.

\subsection{Globalement, l'activité est en avance sur la raison}

L'attention sur l'importance de l'émotion dans la prise de décision a été attirée par l'observation de malades présentant une destruction d'une zone du cortex préfrontal (le cortex préfontal ventromédian). Cette lésion ne perturbait ni leurs résultats aux tests de mesure de l'intelligence, ni leur attention, ni leur maîtrise du langage, ni leur usage de la logique, ni leurs capacités d'apprentissage. Et pourtant, ces personnes s'avéraient incapables de prendre les bonnes décisions dans des situations où elles semblaient pourtant disposer de l'ensemble des notions nécessaires. Ces patients étaient très perturbés dans leurs activités personnelles et sociales. L'explication réside dans les relations qu'entretient le cortex préfrontal avec les structures sous-corticales responsables des réactions émotionnelles (amygdales, hypothalamus). Ces relations permettent de construire, au fil de l'expérience, des liens entre certaines classes de situations complexes et les états émotionnels qu'elles génèrent. Ces liens, gardés en mémoire, donnent leur coloration favorable ou défavorable aux différentes options possibles lorsque se présente un type de situation déjà expérimenté. C'est à partir de ces informations, et non sur la base du raisonnement, qu'un mode de réponse est privilégié. Lorsque, comme chez les malades, le cortex préfrontal ne dispose plus de la mémoire des expériences émotionnelles antérieures, le déclenchement des processus sensorimoteurs 
qui vont aboutir à l'action et à la décision consciente devient dysfonctionnel. Le sujet peut bien connaître les règles qu'il devrait appliquer, celles-ci ne sont pas activées au moment d'agir. Il se voit engagé dans des actions qu'il n'entreprendrait pas si, comme le voudrait le sens commun, sa décision était subordonnée à ses capacités de raisonnement (Damasio, 1995a; 1995b; 1998; 2003).

25 À l'inverse, Béchara a montré que les sujets normaux étaient capables de choisir les stratégies gagnantes avant d'avoir compris que lesdites stratégies étaient effectivement gagnantes, donc avant d'avoir compris quelles règles ils devaient appliquer. Cette performance est due au fait que la décision n'est pas prise sur la base du raisonnement, mais qu'elle est orientée par les expériences émotionnelles antérieures (Bechara, 1997).

Ce rapport aux émotions fait du cerveau un dispositif d'intégration de l'expérience plus performant que la réflexion consciente et en avance sur elle. C'est ce qui explique que le sens clinique du praticien chevronné donne des diagnostics plus pertinents que le système expert. Frith affirme que cette capacité d'intégration de l'expérience fait du cerveau un observateur bayesien ${ }^{1}$ idéal (Frith, 2010).

\subsection{Une volonté déterminée}

27 En somme, le cerveau réagit à des sollicitations de l'environnement dont le sujet n'est que partiellement conscient ; sous l'effet de l'impact émotionnel des expériences antérieures, il libère les dispositions à l'action stabilisées par l'apprentissage ; cet état physiologique est à l'origine de la décision consciente et du déclenchement de l'action. Ces résultats, qui contredisent le sens commun, avaient été anticipés depuis longtemps par certains philosophes. Spinoza est, en la matière, un auteur incontournable :

«Les hommes se trompent en ce qu'ils se croient libres; et cette opinion consiste en cela seul qu'ils ont conscience de leurs actions et sont ignorants des causes par où ils sont déterminés; ce qui constitue donc leur idée de la liberté c'est qu'ils ne connaissent aucune cause à leurs actions. Pour ce qu'ils disent en effet : que les actions humaines dépendent de la volonté, ce sont des mots auxquels ne correspond aucune idée. Car tous ignorent ce que peut être la volonté et comment elle peut mouvoir le corps. » (Spinoza, 1677).

Voltaire aboutit au même constat sur la liberté de la volonté :

«Or, vous recevez toutes vos idées; vous recevez donc votre vouloir, vous voulez donc nécessairement: le mot de liberté n'appartient donc en aucune manière à la volonté. Vous me demandez comment le penser et le vouloir se forment en vous. Je vous réponds que je n'en sais rien. Je ne sais pas plus comment on fait des idées, que je ne sais comment le monde a été fait. Il ne nous est donné que de chercher à tâtons ce qui se passe dans notre incompréhensible machine. La volonté n'est donc point une faculté qu'on puisse appeler libre. » (Voltaire, 1764).

Il faudrait évidemment citer Freud, mais l'obscurité de l'action que nous évoquons ici, dans le contexte d'une approche du travail, ne relève pas de l'inconscient dynamique de la psychanalyse. Les conflits dont nous tentons de rendre compte ne sont pas de l'ordre du sexuel. Nous faisons référence à des dimensions de l'activité qui sont accessibles à la prise de conscience. Notre ambition est donc limitée. Nous estimons cependant que cette distance entre un sujet qui se conçoit comme animé d'une libre volonté et le constat d'une activité dont la logique et l'initiative échappent en grande partie à sa conscience nous introduit à la question de la subjectivité. 


\section{Le retour réflexif sur l'activité : la subjectivité}

\subsection{Illusion d'agentivité et responsabilité}

Wegner une illusion liée à ce qu'il considère comme une sorte d'émotion : une émotion d' agentivité authorship qui accompagne l'action (Wegner, 2004). Il affirme cependant l'importance de cette illusion. En nous faisant ressentir de l'intérieur que ces actions sont les nôtres, elle fournit le composant élémentaire de la psychologie humaine et de la vie sociale. Le sujet voit le résultat de son action; celle-ci s'est accompagnée de la conscience d'une intention, elle a mis en œuvre un mode de réponse dont il sait bien qu'il ne lui est pas étranger et où il retrouve la marque de son histoire et de sa sensibilité ; elle est donc bien sienne, elle engage sa responsabilité.

31 La plupart du temps, le sujet n'éprouve aucun malaise et donc aucun besoin de revenir sur le pourquoi et le comment de son action. Il en va tout autrement lorsque, constatant le résultat et le déplorant, il s'interroge : «Comment ai-je pu faire ou dire cela? » C'est aussi le cas lorsque l'action suscite, de la part d'autrui, des interrogations ou des critiques. Dans les deux cas, le retour interrogateur sur sa propre action et l'effort d'élaboration qu'il impose sont la manifestation de la subjectivité, la manifestation de ce «je » qui doit endosser la responsabilité d'une action dont il n'est pas la cause. Le domaine de la subjectivité au travail, tel que nous l'entendons ici, correspond donc à l'exigence de travail psychique imposé au travailleur par le constat de la distance entre le résultat de son action d'une part, et ses aspirations et/ou les attentes d'autrui d'autre part. Ou bien, sous un angle plus opératoire, l'espace de la subjectivité est ouvert par la distance structurelle entre une activité incarnée et un langage par définition commun, impersonnel, social, par lequel il faut passer pour en rendre compte.

Produire une parole qui rende compte des impasses de l'activité est un enjeu en matière de santé. L'identité vacille lorsque le sujet n'est plus capable de rendre intelligible ce que son mode d'engagement a de potentiellement partageable au regard de la situation particulière dans laquelle il s'est trouvé. L'exigence de produire un récit intelligible qui rétablisse la continuité biographique et renoue les liens avec la communauté témoigne de ce que Ricœur désigne comme le caractère narratif de l'identité (Ricoeur, 1996a; 1996b; Davezies, 1999; 2004). Le passage par le langage est cependant une opération singulière. Il introduit des éléments d'abstraction et de généralité. C'est cette prise de distance avec le vécu immédiat qui permet de partager avec autrui, de confronter les expériences et d'en tirer des leçons pour l'avenir (Vygotski, 1997). Mais dans ce cas, comment l'élaboration langagière peut-elle donc avoir une efficacité ? Elle nous conduit dans le monde des idées, or c'est au niveau de l'action que se situe le conflit et nous savons maintenant que l'idée n'est pas la cause de l'action. Pour remplir sa fonction d'outil au service du développement, le langage et la pensée qu'il véhicule doivent exercer un pouvoir de détermination sur l'activité.

\subsection{L'enracinement corporel du langage}

33 Là encore, la physiologie nous propose des éléments de réponse. L'idée d'un langage manipulant de pures idéalités symboliques est de plus en plus mise en doute. Un nombre

Perspectives interdisciplinaires sur le travail et la santé, 14-2 | 2012 
croissant de recherches insistent sur son lien avec le vécu des situations et sur son caractère incarné. La compréhension d'une phrase dépend de la capacité à simuler intérieurement le rapport du sujet à l'état du monde que la phrase décrit (Jirak, 2010). Les mots évoquant une odeur activent les aires cérébrales responsables de l'olfaction, la présentation écrite de mots évoquant des sons active les aires auditives. Surtout, la compréhension des verbes d'action active, dans les aires prémotrices et motrices, les zones correspondant au mouvement évoqué. Cette simulation motrice n'accompagne pas le traitement sémantique de la parole, elle constitue le processus sémantique lui-même (Pulvermüller, 2010 ; Knoeferle, 2010). Il est d'ailleurs possible de mettre en évidence les effets comportementaux des interactions entre le langage et le mouvement: la compréhension d'un terme évoquant un mouvement et la réalisation d'un mouvement sont susceptibles de mobiliser des ressources neurales communes, avec, selon les cas, des effets réciproques de facilitation ou de perturbation (Feldman, 2010). Ces résultats sont confirmés par diverses approches: la stimulation transcranienne des zones motrices corticales accélère les tâches de traitement syntaxique des verbes d'action (Pulvermüller, 2005) ; à l'inverse, les malades présentant une pathologie de la motricité (parkinson, sclérose latérale amyotrophique) voient leurs performances réduites dans le domaine du traitement des verbes d'action (Bak, 2004; Boulenger, 2008). Évoquer l'action, c'est la simuler intérieurement. Une conversation n'est donc pas un pur échange symbolique. L'intercompréhension implique qu'à la configuration sensitivomotrice activée, dans le cerveau du locuteur par l'évocation d'une situation ou d'une action, réponde l'activation d'une configuration sensitivomotrice réalisant une simulation interne de la situation et de l'action dans le cerveau de l'auditeur. L'échange langagier ne met pas seulement le concept en relation avec le concept, il fait circuler des configurations sensorimotrices entre les interlocuteurs. Il transmet donc, dans le même mouvement, des idées et des dispositions corporelles à l'action. À la prochaine occasion, l'activité se déploiera à nouveau sans attendre la réflexion, mais à partir d'un répertoire de dispositions à l'action enrichi par la discussion.

L'usage du langage au cours d'une conversation présente d'ailleurs les mêmes caractéristiques de spontanéité que celles observées dans le cas de l'action: les zones cérébrales qui sont mobilisées par la prise de parole sont activées avant que le sujet n'ait pris la décision d'intervenir. L'intention de parler comme l'intention d'agir trouvent leurs origines, à un stade encore non conscient, dans les mêmes aires cérébrales (du cortex pariétal) (Carota, 2010).

En somme, nous décrivons deux moments décisifs: l'activation, sous l'impulsion des sollicitations de la situation, des dispositions à l'action préalablement incorporées, et un développement de ces dispositions sous l'effet du retour réflexif sur l'activité grâce à la médiation du langage. Dans les deux cas, le sujet apparait porté par la dynamique de l'activité beaucoup plus qu'il ne l'amorce Une telle analyse ne laisse que peu de place à la pensée comme réalité autonome.

\subsection{Activité et pensée}

Contre l'idée d'une dépendance de la pensée vis-à-vis de l'activité, on pourra objecter, avec raison, qu'il y a des idées qui ne correspondent pas à des expériences vécues et qui ne sont donc pas susceptibles d'activer des configurations sensitivomotrices. Il y a, en effet, des mots abstraits dont le sens ne tient qu'à leurs liens avec d'autres mots abstraits. 
Nous pouvons cependant, ici encore, relever des proximités entre la dynamique de l'action, telle que la décrit la physiologie, et celle de la pensée. Autant l'idée selon laquelle l'action ne serait pas causée par la volonté heurte le sens commun, autant l'indépendance de la pensée par rapport à la volonté relève de l'expérience commune. Nietzsche a exprimé de façon très vive cette spontanéité de la pensée :

«Qu'est-ce qui me donne le droit de parler d'un «je » et d'un «je » qui soit une cause, et pour comble, cause de la pensée. [...] Je ne me lasserai jamais de souligner un petit fait très bref que les gens atteints de superstition n'aiment guère avouer ; c'est à savoir qu'une pensée vient quand " elle » veut et non quand «je » veux, en telle sorte que c'est falsifier les faits que de dire que le sujet «je» est la détermination du verbe "pense ». Quelque chose pense, mais que ce soit justement ce vieil et illustre "je», ce n'est là, pour le dire en termes modérés, qu'une hypothèse, une allégation " (Nietzsche, 1886).

d'incorporation qui ont mis en place les ressources intérieures. Naccache décrit ce processus pour la recherche mathématique qui apparait pourtant comme le domaine par excellence de la pensée abstraite (Naccache, 2009). La découverte géniale est le résultat d'un cheminement en plusieurs étapes. La première consiste en un travail d'explicitation approfondie de la nature du problème. Elle implique une mobilisation consciente. Il s'agit même d'un moment pénible marqué par «une intense sensation d'effort mental». Ce travail est décisif, il réalise le processus d'incorporation, mais il ne donne pas la solution. La seconde phase est dite d'incubation, le chercheur vaque à d'autres occupations, mais son cerveau continue à travailler de façon inconsciente sur le problème à partir du matériau accumulé. Enfin l'idée géniale jaillit - Archimède bondit hors de son bain. Mais cette spontanéité de l'idée ne doit pas masquer le fait que l'idée ne serait pas survenue si un travail préalable d'explicitation approfondie n'avait pas été accompli. Et il faudra encore tout un travail de réajustement des conceptions initiales sous l'effet de la découverte pour que celle-ci porte ses fruits.

L'action comme la pensée ne sont donc pas causées par l'exercice d'une volonté libre qui trouverait en elle-même les ressources de son mouvement. En revanche, leur développement, c'est-à-dire leur capacité à ouvrir de nouveaux espaces, dépend étroitement du travail de réflexion qui, préalablement, a permis l'incorporation des éléments conceptuels véhiculés par le langage. Ceux-ci seront ensuite mobilisés selon des processus non conscients pour résoudre le problème. La pensée est donc produite par un processus inconscient, mais celui-ci repose sur la mobilisation de ressources incorporées qui doivent avoir, à un moment ou un autre, fait l'objet d'une prise de conscience et donc d'une réflexion (Naccache, 2009).

\subsection{Une pensée en situation}

40 Le processus d'incorporation suppose d'enrichir la représentation du problème en l'envisageant de la façon la plus concrète et sous une multiplicité de points de vue; il ne peut pas être renvoyé au seul dialogue intérieur de l'individu isolé, en raison même de l'obscurité que revêt son activité à ses propres yeux. La confrontation à l'expérience d'autrui est un moment décisif de la prise de conscience. Dans le cas du chercheur, elle 
passe par la lecture des publications scientifiques; dans le cas des dilemmes rencontrés au quotidien dans le travail, la ressource se situe du côté d'une discussion qui s'efforcerait de serrer au plus près les dilemmes de l'activité.

Un processus de pensée abstrait ne peut pas réellement produire de la compréhension s'il ne revient pas à la pratique, c'est-à-dire au vécu corporel. En effet, comprendre un concept, c'est comprendre ce qu'il change dans notre façon de percevoir le réel. Il n'y a pas de signification qui ne se ramène à des effets pratiques dans telle ou telle situation. Ce principe a été formulé par Peirce, penseur majeur de la philosophie américaine, logicien et père de la sémiotique. Il est condensé dans une maxime célèbre :

« Considérer quels sont les effets pratiques que nous pensons pouvoir être produits par l'objet de notre conception. La conception de tous ces effets est la conception complète de l'objet. » (Peirce, 1879).

La compréhension d'une notion n'est rien d'autre que la capacité à penser - et nous ajouterons donc à simuler intérieurement - ses effets pratiques. À l'inverse, si le discours reste dans le domaine de l'abstraction, l'idée n'explique pas grand-chose et n'a pas d'effet sur l'action.

Nous insistons sur ce point car, de tous côtés, nous rencontrons la tentation de ramener les problèmes du rapport subjectif au travail à des généralités abstraites telles que le niveau d'exigence, le manque d'autonomie, le défaut de reconnaissance, etc., sans plus se préoccuper de la signification pratique de ces notions. Il y a, derrière ces problématiques, une surestimation de la capacité des idées à produire des effets sans un travail considérable de contextualisation et d'incorporation des enjeux concrets en matière d'action. Dewey, un des théoriciens américains de la démocratie, exprime un jugement sévère contre les discours qui prétendent se déployer à l'abri de cette exigence du retour aux situations et à la pratique :
« Nous vivons et nous agissons en connexion avec l'environnement existant, non en connexion avec des objets isolés, [...] Le discours qui n'est pas contrôlé par référence à une situation n'est pas un discours, mais un galimatias sans signification, exactement comme un ensemble de caractères d'imprimerie ne fait pas une ligne linotypée, encore moins une phrase. L'univers de l'expérience est la précondition de l'univers du discours. Sans le contrôle de l'univers de l'expérience, il n'y a aucun moyen de déterminer la convenance, le poids ou la cohérence d'une distinction ou d'une relation donnée. [...] La présence d'une situation à titre d'univers du discours apparaît comme la condition englobante et régulatrice de tout discours. » (Dewey, 1993).

Le retour réflexif sur l'activité est donc un enjeu central du développement humain, individuel et collectif. Sans la médiation du langage comme lien entre l'activité personnelle et celle d'autrui, l'activité et la vie psychique sont vouées à la répétition, au repli et à la désolation. Mais, compte tenu de ce que nous avons exposé, on comprendra qu'il ne suffit pas de se mettre autour d'une table pour que s'exprime l'expérience du travail dans le débat social au sein de l'entreprise. Avant l'étape de la discussion avec la hiérarchie, l'expérience doit faire l'objet d'une élaboration. Celle-ci exige la mise en place de cadres adéquats. 


\section{Un cadre pour l'élaboration}

\subsection{La prise en charge du sujet souffrant : une affaire en général mal engagée}

À l'origine de la souffrance au travail, il y a toujours un désaccord sur la façon de travailler. Si l'accent est mis sur la seule dimension interpersonnelle, c'est parce que ce désaccord se présente dans un contexte relationnel déséquilibré. Dans le scénario type, le supérieur met en avant une défaillance sur tel ou tel point. Dans ce mouvement, il met l'accent sur un critère de performance qui lui importe, mais il ne se met pas personnellement en jeu ; le travailleur, au contraire, est interpellé dans son activité. Pour que le conflit se développe sous la forme d'un débat susceptible de produire une évolution positive, il faudrait que le salarié puisse revenir sur son activité afin d'expliciter les enjeux qui ne sont pas perceptibles à partir de la position du chef, mais sont néanmoins pertinents au regard de son expérience professionnelle. Or, il ne dispose ni des temps, ni des espaces de discussion nécessaires à cette élaboration. De ce fait, il est en position de se sentir attaqué dans ce qu'il est. Il vit la critique sur le mode intrusif, persécutoire. Une fois engagée, cette réaction est souvent encouragée par l'entourage et structurée grâce aux éléments de langage sur le harcèlement moral qui circulent dans le monde du travail et sont proposés comme un mode d'expression de la souffrance susceptible de pallier le déficit d'élaboration. La conséquence en est souvent une radicalisation d'un conflit dans lequel le salarié a de grandes chances d'être perdant. La situation de stress chronique ainsi créée est responsable d'une augmentation de la sécrétion de cortisol dont l'action prolongée sur l'hippocampe (une structure cérébrale du lobe temporal) perturbe la capacité à recontextualiser le problème. Il devient de plus en plus difficile de reprendre la main sur la situation. La tendance à la surgénéralisation pousse le sujet vers la dépression (Davezies, 2004; 2009).

Dans les cas moins dramatiques, l'intelligibilité de la situation est recherchée du côté des facteurs de risques psychosociaux: manque d'autonomie, isolement, défaut de reconnaissance... Alors que le conflit témoigne d'un à venir qui insiste dans la situation, sollicite l'activité et appelle de nouvelles réponses, le diagnostic ramène au passé, aux discours stabilisés, aux connaissances acquises. L'analyse passe ainsi à côté de l'exigence de renouvellement qui tend la situation.

47 Enfin, le professionnel convaincu de la nécessité d'un ancrage de l'analyse dans la situation, comme seul moyen d'ouvrir des perspectives nouvelles, peut s'interroger : doiton croire ce que dit le salarié ? Comment démêler le vrai du faux ? Cette question laisse entendre que le problème pour le clinicien serait d'adhérer ou non à l'analyse que le travailleur fait de sa situation. Or, si ce dernier demande de l'aide et si la situation menace sa santé mentale, c'est justement parce qu'il n'arrive à penser qu'une part très réduite de l'impasse dans laquelle il est enfermé. Coller au discours de la victime ne peut que l'enfoncer dans les difficultés dont elle tente de sortir. Il faut garder en tête le caractère énigmatique du travail aux yeux même de celui qui l'exécute. Si l'on admet le caractère interprétatif et pour partie fictionnel de la conscience (Naccache, 2009), alors la question n'est pas de croire ou de ne pas croire. L'assistance consiste à aider le salarié à revenir sur son activité et à mieux prendre conscience de ses enjeux et de leurs dimensions conflictuelles. Non pas expliquer au salarié ce qu'il vit, mais se faire expliquer 
ce qui se passe, au plus près des situations concrètes. L'avantage de cette position d'interrogation sur le travail est qu'elle peut être tenue tout aussi fermement face au salarié en difficulté, face à son chef ou face au patron, car la conscience de l'employeur n'est pas moins interprétative et fictionnelle que celle du salarié de base.

Une façon de mener l'enquête peut consister à ne pas se focaliser d'emblée sur les derniers événements qui sont généralement saturés d'enjeux affectifs et, de ce fait, difficiles à démêler. Revenir à la période antérieure, au moment où les choses se passaient bien permet de poser des questions sur ce qui plaisait, ce qui mobilisait, ce qui posait problème. Évidemment, l'expression du travailleur est une reconstruction à la lumière de l'expérience actuelle mais, de ce fait, elle jette souvent un premier éclairage sur ses enjeux. Ensuite, il y a eu les premiers incidents qui, souvent, ont surpris le travailleur, sans qu'il soit en mesure d'en évaluer clairement la dynamique. La charge affective de ces incidents est plus réduite, il est intéressant d'y revenir, car il sera plus facile pour le sujet d'analyser ce qui, à l'époque, à ces occasions, s'est manifesté comme divergence dans la façon de donner priorité aux enjeux du travail. Il est ainsi possible de reprendre les divers événements qui ont marqué la dégradation de la relation jusqu'à en arriver à la situation critique qui motive l'enquête. Il restera bien un conflit interpersonnel, mais ramené à des enjeux de travail intelligibles, et donc susceptibles d'être expliqués et partagés avec autrui (Davezies, 1999; 2009).

Ce travail a un effet en matière de santé. Mieux comprendre la dynamique du conflit dans lequel il est engagé offre au salarié des capacités nouvelles pour expliciter sa position face à autrui et la défendre. Il récupère ainsi une capacité à agir qui constitue une protection de sa santé (Davezies, 2008). Sous le conflit interpersonnel, l'analyse révèle les contradictions de l'organisation du travail. Le travail de prise en charge est accompli lorsqu'une question d'organisation du travail est renvoyée au débat social de la façon la plus concrète, alors que, jusque-là, elle était portée par l'individu souffrant comme une question personnelle.

\subsection{Le retour à la discussion collective}

50 Au-delà de la prise en charge individuelle des personnes en difficulté, il apparaît que le travail impose aux individus des arbitrages serrés, que ce phénomène est à l'origine d'une montée des conflits (Béroud, 2008), et que les salariés abordent ceux-ci dans une position très défavorable, faute d'espaces d'élaboration sur les enjeux de leur activité. Dans ce contexte, la prise en charge individuelle ne constitue qu'un palliatif. La montée de la souffrance au travail appelle la construction d'espaces de discussion collective où la confrontation de chacun à l'activité et à l'expérience d'autrui puisse contribuer au développement individuel et collectif. Seulement, en amont des espaces d'expression qui permettent la discussion avec la hiérarchie, les salariés ont besoin d'espaces d'élaboration autonomes.

51 L'idée d'une élaboration hors de la présence de la hiérarchie peut choquer. Et pourtant, il n'est pas raisonnable de demander à celle-ci d'animer et de soutenir l'élaboration de points de vue du personnel avec lesquels elle devra composer. C'est d'autant plus vrai dans le contexte actuel où la hiérarchie a beaucoup de mal à faire entendre ses propres difficultés à sa direction. Compte tenu de la mission qui lui est dévolue, le délégué du personnel serait plus en position d'animer la discussion, à la condition qu'il bénéficie d'une formation et d'une possibilité de soutien en matière d'analyse du travail. Car il ne 
s'agit pas ici de faire œuvre de psychologue. Il faut simplement mettre collectivement des mots sur les enjeux de l'activité en s'efforçant d'aborder celle-ci avec un grain plus fin que celui des discours habituels.

52 La hiérarchie elle-même pourrait y trouver un intérêt. En situation de crise, les cadres et la maîtrise sont incités par les directions à mieux écouter le personnel. Mais, il leur est bien difficile de faire quelque chose à partir de cette position d'écoute, dans un contexte où les agents ne sont pas d'accord entre eux et ne se retrouvent que sur des questions très générales qui dépassent la capacité d'intervention du supérieur hiérarchique. Il n'est donc pas sûr que la hiérarchie serait fragilisée dans une situation où les agents seraient mieux à même d'élaborer et d'exprimer leurs points de vue sur le travail. Les recherchesactions menées dans cette perspective avec des syndicalistes (Théry, 2006; Chassaing, 2010) montrent que l'élaboration des questions au plus près du travail ouvre des perspectives nouvelles et change les conditions du débat social en rétablissant l'autorité potentielle que confère aux salariés leur expérience du travail (Davezies, 2010; Gache, 2012).

\section{Prolongement : le besoin d'une réorientation des analyses en santé au travail}

53 Tous les éléments que nous avons évoqués conduisent à interroger la problématique traditionnelle en matière de santé au travail. Qu'elles soient menées par les services de santé au travail ou par les cabinets de consultants, toutes les interventions ont un but: informer la direction, enrichir son point de vue, l'aider à prendre des décisions moins inutilement coûteuses, la rendre plus intelligente, en somme. Du côté des représentants du personnel ou de l'expertise CHSCT, la problématique est au fond la même: le destinataire des discours et analyses est la direction. Or, la montée en puissance du thème de la souffrance au travail souligne qu'une telle problématique saute une étape majeure. Le premier niveau de problème se situe du côté de l'élaboration et de l'expression de l'expérience du travail. Le déficit d'élaboration fragilise les individus, il participe au déséquilibre des processus de décision au bénéfice des indicateurs statistiques, comptables, financiers, et il passe à côté des potentiels de transformation que recèle la dynamique de l'activité. D'une façon générale, l'accent est mis sur la tension entre les discours du personnel et ceux de la direction, alors que l'origine de la souffrance, mais aussi les ressources pour transformer la situation, sont du côté de la tension entre ce que font les agents et les formes langagières dont ils disposent pour en rendre compte. Les premiers destinataires des constats et analyses du travail devraient donc être les travailleurs eux-mêmes, non pas seulement pour recueillir leurs points de vue ou pour valider l'analyse, mais comme moyen de soutenir et développer leur propre réflexion sur les enjeux de leur activité. L'expérience prouve qu'à peu près toute investigation sur le travail peut être utile si elle n'est pas considérée comme un aboutissement qu'il serait urgent de présenter à la direction, mais comme une base à partir de laquelle il est possible d'aider les salariés à clarifier, au plus près des situations, les enjeux des conflits qui se nouent autour des objets du travail : conflits avec la direction, mais aussi, et peutêtre avant tout, conflits entre les salariés eux-mêmes. Un tel travail, attentif à ce que les discussions n'occultent pas les différences entre individus, permet de porter la réflexion et l'activité à un niveau supérieur. Il s'agirait, en somme, d'organiser, entre les travailleurs, ce que Clot a mis en avant sous le terme de dispute (Clot, 2008, 2010), en 
référence au sens noble que pouvait avoir la disputatio dans la scolastique médiévale. La nécessaire discussion des agents avec leur hiérarchie ne pourrait que s'en trouver enrichie. La perspective peut paraître ambitieuse, mais la situation actuelle dans laquelle une foule de professionnels extérieurs (consultants, services de santé au travail, services sociaux, etc.) s'interposent entre salariés et directions pour pallier un déficit de discussion témoigne d'une situation sociale franchement pathologique.

\section{BIBLIOGRAPHIE}

Aarts, H., Chartrand, T.L., Custers, R., Danner, U., Dik, G., Jefferis, V.E., Cheng, C.M. (2005). Social Stereotypes and Automatic Goal Pursuit. Social Cognition, 23, 6, 465-490.

Bak, T.H., Hodges, J.R. (2004). The effects of motor neurone disease on language: further evidence. Brain and Langage, 89, 2, 354-61.

Bargh, J.A., Gollwitzer, P.M., Lee-Chai, A., Barndollar, K., Trötschel, R. (2001). The automated will: nonconscious activation and pursuit of behavioral goals. Journal of Personality and Social Psychology , 81, 6, 1014-1027.

Bechara, A., Damasio, H., Tranel, D., Damasio, A.R. (1997). Deciding advantageously before knowing the advantageous strategy. Science, 28, 5304, 1293-5.

Beroud, S., Demis, J.M., Desage, G., Pelisse, J. (2008). Entre grèves et conflits : les luttes quotidiennes au travail. Rapport de recherche ${ }^{\circ} 49$. Centre d'études de l'emploi, Paris.

Boulenger, V., Mechtouff, L., Thobois, S., Broussolle, E., Jeannerod, M., Nazir, T.A. (2008). Word processing in Parkinson's disease is impaired for action verbs but not for concrete nouns. Neuropsychologia, 31;46, 2, 743-56.

Carota, F., Posada, A., Harquel, S., Delpuech, C., Bertrand, O., Sirigu, A. (2010). Neural dynamics of the intention to speak. Cerebral Cortex, 20, 8, 1891-7.

Chambre de commerce et d'industrie de Paris (2007). Le management participatif: source d'économie et de renforcement de la compétitivité de l'entreprise. Les ressources humaines en pratique, p. 30. www.ccip75.fr/upload/pdf/capRH30_Tendance.pdf

Chassaing, K., Daniellou, F., Davezies, Ph., Duraffourg, J., Bongiorno, Y., Dufour, S., Gâche, F., Lusson, J. (2010). Prévenir les risques psychosociaux dans l'industrie automobile : élaboration d'une méthode d'action syndicale. Rapport IRES, Émergences.

Clot, Y. (2010). Le travail à cœur. Pour en finir avec les risques psychosociaux. Éditions La Découverte, Paris.

Clot, Y. (2008). Travail et pouvoir d'agir. PUF, Paris.

Custers, R., Maas, H., Wildenbeest, M., Aars, H. (2008). Nonconscious goal pursuit and the surmounting of physical and social obstacles. European Journal of Social Psychology, 38, 1013-1022.

Custers, R., Aarts, H. (2010). The unconscious will: how the pursuit of goals operates outside of conscious awareness. Science, 329, 5987, 47-50. 
Damasio, AR. (1995). On some functions of the human prefrontal cortex. Annals of the New York Academy of Sciences, 15, 769:241-51.

Damasio, A.R. (2003). Spinoza avait raison : joie et tristesse, le cerveau des émotions. Paris: Odile Jacob.

Damasio, A.R. (1998). Emotion in the perspective of an integrated nervous system. Brain Research Reviews, 26, 2/3, 83-6.

Damasio, A.R. (1995). L'erreur de Descartes : La raison des émotions. Odile Jacob, Paris.

Danckert, J., Rossetti ,Y. (2005). Blindsight in action: what can the different sub-types of blindsight tell us about the control of visually guided actions? Neuroscience \& Biobehavioral Reviews , 29, 7, 1035-46.

Daprati, E., Sirigu, A., Nico, D. (2010). Body movement: consciousness in the parietal lobes. Neuropsychologia, 48, 756-762.

Davezies, P. (2010). Sur le rapport à l'autorité en clinique du travail. Pouvoir d'agir et autorité dans le travail. Ed. F. Hubault, p. 31-40, Octares Éditions, Toulouse. http://philippe.davezies.free.fr/ download/down/Autorite_travail_2008.pdf

Davezies, P. (2009). Souffrance au travail : comprendre avant d'agir. Santé et travail, 68. http:// philippe.davezies.free.fr/download/down/2009_Comprendre.pdf

Davezies, P. (2008). Stress, pouvoir d'agir et santé mentale. Archives des Maladies Professionnelles, 69, 195-203.

Davezies, P., Deveaux, A., Torres, C. (2006). Repères pour une clinique médicale du travail. Archives des Maladies Professionnelles, 67, 119-125.

Davezies, P. (2005). Souffrance au travail, le risque organisationnel. Journées médicales du CISME sur le risque organisationnel. http://philippe.davezies.free.fr

Davezies, P. (2004). Les impasses du harcèlement moral. Travailler, 11, 83-90. http:// philippe.davezies.free.fr/download/down/Harcelement.rtf.pdf

Davezies, P. (1999). Transformations des organisations du travail, nouvelles pathologies : défis à la clinique médicale. Archives des Maladies Professionnelles, 60, 6, 542-550.

Desmurget, M., Sirigu, A. (2009). A parietal-premotor network for movement intention and motor awareness. Trends in Cognitive Sciences, 13, 10, 411-9.

Dewey, J. (1993). Logique, la théorie de l'enquête. PUF, Paris.

Elovainio, M., Ferrie, J.E., Gimeno, D., Devogli, R., Shipley, M., Vahtera, J., Brunner, E., Marmot, M.G., Kivimäki, M. (2010). Organizational justice and markers of inflammation: The Whitehall II study. Occupational and Environmental Medicine, 67, 2, 78-83.

Elovainio, M., Ferrie, J.E., Gimeno, D., de Vogli, R., Shipley, M., Brunner, E., Kumari, M., Vahtera, J., Marmot, M.G., Kivimäki, M. (2009). Organizational Justice and Sleeping Problems: The Whitehall II Study. Psychosomatic Medicine, 71, 334-340.

Elovainio, M., Leino-Arjas, P., Vahtera, J., Kivimäki, M. (2006). Justice at work and cardiovascular mortality: a prospective cohort study. Journal of Psychosomatic Research, 61, 2, 271-4.

Elovainio, M., Kivimäki, M., Vahtera, J. (2002). Organizational Justice: Evidence of a New Psychosocial Predictor of Health. American Journal of Public Health, 92, 1, 105-108.

Feldman, J. (2010). Embodied language, best-fit analysis, and formal compositionality. Physics of Life Reviews, 7, 4, 385-410. 
Fitzsimons, G.M., Bargh, J.A. (2003). Thinking of you: Nonconscious pursuit of interpersonal goals associated with relationship partners. Journal of Personality and Social Psychology, 84, 1, 148-164.

Fourneret, P., Jeannerod, M. (1998). Limited conscious monitoring of motor performance in normal subjects. Neuropsychologia, 36, 11, 1133-40.

Fried, I. (2011). Internally generated preactivation of single neurons in human medial frontal cortex predicts volition. Neuron, 69, 548-562.

Frith, C. (2010). The neural basis of hallucinations and delusions. Comptes rendus biologies, 328, 2, $169-75$.

Frith, C. (2010). Comment le cerveau crée notre univers mental. Paris, Odile Jacob.

Gache, F. (2012). Faire du syndicat un outil pour le développement du pouvoir d'agir des salariés. Les leçons d'une recherche-action. Archives des Maladies Professionnelles, 73, 239-245.

Gilbert, S.G., Laschinger, H.K.S., Leiter, M. (2010). The mediating effect of burnout on the relationship between structural empowerment and organizational citizenship behaviours. Journal of Nursing Management, 18, 339-348.

Gimeno, D., Tabák, A.G., Ferrie, J.E., Shipley, M.J., De Vogli, R., Elovainio, M., Vahtera, J., Marmot, M.G., Kivimäk,i M. (2010). Justice at Work and Metabolic Syndrome: the Whitehall II Study. Occupational and Environmental Medicine, 67, 4, 256-62.

Guérin, F., Laville, A., Daniellou, F., Duraffourg, J., Kerguelen, A. (1997). Comprendre le travail pour le transformer. ANACT, Paris.

Haggard, P., Eimer, M. (1999). On the relation between brain potentials and the awareness of voluntary movements. Experimental Brain Research, 126, 128-133.

Haggard, P. (2008). Human volition: towards a neuroscience of will. Nature Reviews Neuroscience, 9, 934-946.

Jeannerod, M. (2009). Le cerveau volontaire. Odile Jacob, Paris.

Jirak, D., Menz, M.M., Buccino, G., Borghi, A.M., Binkofski, F. (2010). Grasping language--a short story on embodiment. Consciousness and Cognition, 19, 3, 711-20.

Karasek, R., Theorell, T. (1990). Stress, productivity and the reconstruction of working life. Library of Congress, $381 \mathrm{p}$.

Knoeferle, P., Crocker, M.W., Pulvermüller, F. (2010). Sentence processing and embodiment. Brain and Language, 112, 3, 137-42.

Kouvonen, A., Vahtera, J., Elovainio, M., Cox, S.J., Cox, T., Linna, A., Virtanen, M., Kivimäki, M. (2007). Organisational justice and smoking: the Finnish Public Sector Study. Journal of Epidemiology and Community Health, 61, 5, 427-33.

Kouvonen, A., Kivimäki, M., Elovainio, M., Väänänen, A., de Vogli, R., Heponiemi, T., Linna, A., Pentti, J., Vahtera, J. (2008). Low organisational justice and heavy drinking: a prospective cohort study. Occupational and Environmental Medecine, 65, 1, 44-50.

Lachmann, H., Larose, C., Penicaud, M. (2010). Bien-être et efficacité au travail - 10 propositions pour améliorer la santé psychologique au travail. La Documentation française. www.dgdr.cnrs.fr/ $\mathrm{drh} /$ protect-soc/documents/fiches_rps/rapport_lachmann.pdf

Libet, B., Gleason, C.E., Wright, E.W., Pearl, D.K. (1983). Time of conscious intention to act in relation to onset of cerebral activity (readiness potential). The unconscious initiation of freely voluntary act. Brain, 106, 23-42. 
Libet, B. (1999). Do we have free will. Journal of Consciousness Studies, 6, 8/9, 47-57.

Malafouris, L. (2008). At the Potters's Wheel: An argument for material agency. In, Material Agency: Toward a non-anthropocentric approach, Eds C. Knappett, L. Malafouris, p. 19-36, Springer Science, New York.

Moorman, R.H. (1991). Relationship between organizational justice and organizational citizenship behaviors: do fairness perceptions influence employees citizenship? Journal of Applied Psychology, 76, 6, 845-855.

Moorman, R.H., Nietoff, B.P., Organ, D.W. (1993). Treating employees fairly and organizational citizenship behavior: sorting the effects of job satisfaction, organizational commitment and procedural justice. Employee Responsibilities and Rights Journal, 6, 3, 209-225.

Naccache, L. (2009). Le nouvel inconscient. Freud, le Christophe Colomb des neurosciences. Odile Jacob, Paris.

Neidhammer, I., Siegrist, J. (1998) Facteurs psychosociaux au travail et maladies cardiovasculaires : l'apport du modèle du Déséquilibre Efforts/Récompenses. Revue d'épidémiologie et de santé publique, 46, 5, 398-410.

Nietzsche, F. (1886). Par-delà le bien et le mal. Éditions Aubier-Montaigne, Paris.

NF ISO 26000 (2010). Lignes directrices relatives à la responsabilité sociétale. AFNOR, Paris.

Organ, D.W. (1990). The motivational basis of organizational behavior. Research in Organizational Behavior, 12, 43-72.

Peirce, C.S. (1879). Comment rendre nos idées claires. Revue philosophique de la France et de l'étranger, VII, 39-57.

Pulvermüller, F. (2005). Brain mechanisms linking language and action. Nature Reviews Neurosciences, 6, 7, 576-82.

Ricoeur, P. (1996). Entre mémoire et histoire. Projets, 248, -16.

Ricoeur, P. (1996). Les paradoxes de l'identité. L'information psychiatrique, 3, 201-206.

Sennet, R. (2010). Ce que sait la main. La culture de l'artisanat. Albin Michel, Paris.

Shergill, S.S., Samson, G., Bays, P.M., Frith, C.D., Wolpert, D.M. (2005). Evidence for sensory prediction deficits in schizophrenia. American Journal of Psychiatry, 162, 12, 2384-6.

Pulvermüller, F., Fadiga, L. (2010). Active perception: sensorimotor circuits as a cortical basis for language. Nature Reviews Neurosciences, 11, 5, 351-60.

Sirigu, A., Daprati, E., Ciancia, S., Giraux, P., Nighoghossian, N., Posada, A., Haggard, P. (2004). Altered awareness of voluntary action after damage to the parietal cortex. Nat, Neurosci., 7, 1, 80-84.

Smith, C.A., Organ, D.W. (1983). Near JP. Organizational citizenship behavior: Its nature and antecedents. J. Appl. Psychol., 68, 4, 653-663.

Soon, C.S., Brass, M., Heinze, H.J., Haynes ,J.D. (2008). Unconscious determinants of free decisions in the human brain. Nature Neuroscience, 11, 543-545.

Spinoza, B. (1677). Éthique. Éditions Garnier Frères, Paris.

Strahan, E.J., Spencer, S.J., Zanna, M.P. (2002). Subliminal priming and persuasion: Striking while the iron is hot. Journal of Experimental Social Psychology, 38, 6, 556-568 
Théry, L. (2006). Le travail intenable. Résister collectivement à l'intensification du travail. La Découverte, Paris.

Voltaire (1819). Cuvres complètes, dictionnaire philosophique, tome IV. Armand-Aubrée Éditeurs, Paris.

Von, Holst E., Mittelstaedt, H. (1980). The reafference principle - Interaction between the central nervous system and the periphery. In, The Organization of action: a new synthesis, Ed. Gallistel C.R., p. 176-209, Lawrence Erlbaum Associates, Hillsdale.

Vygotski, L. (1997). Pensée et langage. La Dispute/SNEDIT, Paris.

Wegner, D.M. (2004). Précis of the illusion of conscious will. Behavioral and Brain Sciences, 27, 5, 649-59.

Weiskrantz, L., Warrington, E.K., Sanders, M.D., Marshal, J. (1974). Visual capacity in the hemianoptic field following a restricted occipital ablation. Brain, 97, 709-728.

Ylipaavalniemi, J., Kivimäki, M., Elovainio, M., Virtanen, M., Keltikangas-Järvinen, L., Vahtera, J. (2005). Psychosocial work characteristics and incidence of newly diagnosed depression: a prospective cohort study of three different models. Social Sciences and Medicine, 61, 1, 111-22.

\section{NOTES}

1. La statistique bayésienne vise à estimer la probabilité qu'un signe ou un ensemble de signes soit l'indice d'une situation type connue. Elle est à la base du fonctionnement du système expert.

\section{RÉSUMÉS}

L'intensification du travail oblige les salariés de tous secteurs et de tous niveaux hiérarchiques à des arbitrages serrés entre les différents objectifs du travail. Faute d'espace de discussion, chacun se débrouille seul, avec comme conséquence une individualisation des questions, une montée des conflits et une dégradation de la performance. Cette situation appelle la mise en place des espaces d'expression sur le travail, prévus dans la loi française. Cette orientation se heurte cependant à une difficulté : l'activité du travailleur est en avance sur sa conscience et beaucoup plus riche que ce qu'il est capable d'exprimer. Des espaces autonomes d'élaboration des enjeux du travail sont nécessaires pour créer les conditions d'une discussion avec la hiérarchie susceptible de porter ses fruits dans le domaine de la santé au travail et dans celui de la performance globale de l'entreprise.

Regardless of the job sector or hierarchical position, work intensification forces workers to make hard choices between the different goals they are expected to meet. Due to a lack of discussion, people have to manage by themselves. The consequences are a rising tide of interpersonal conflicts and occupational stress associated with a deterioration in overall performance. This situation requires that time and places be set aside for work disputes, in keeping with French law. Nevertheless, there is a serious difficulty in actualizing this law: the worker's tacit knowledge of his work is much richer than his conscious ability to verbalize and describe the 
work activity. Self-governed, employee-reserved times and places for working out the stakes at issue are necessary before discussing with the administration if we are to arrive at favourable results for occupational health and overall company performance.

La intensificación del trabajo obliga a los empleados, independientemente del sector o del nivel jerárquico al que pertenezcan, a un arbitraje difícil entre los diferentes objetivos del trabajo. Falta de espacios de discusión, cada cual de desenvuelve solo, lo que tiene como consecuencia una individualización, un aumento de los conflictos interpersonales y una degradación del rendimiento laboral. Esta situación requiere que se creen espacios de expresión sobre el trabajo según estipula la ley francesa. No obstante, esta orientación se enfrenta a una dificultad: la actividad del trabajador es anterior a su consciencia y mucho más rica que lo que el trabajador puede expresar. Por lo tanto, espacios autónomos de elaboración de las cuestiones del trabajo son necesarios para crear las condiciones de una discusión con los niveles jerárquicos. Discusión que sea fructífera desde el punto de vista de la salud laboral y del rendimiento global de la empresa.

\section{INDEX}

Keywords : occupational stress, activity, subjectivity, neurosciences

Mots-clés : souffrance au travail, activité, subjectivité, neurosciences

Palabras claves : sufrimiento en el trabajo, actividad, subjetividad, neurociencias

\section{AUTEUR}

\section{PHILIPPE DAVEZIES}

Laboratoire de médecine et santé au travail, Université Claude Bernard Lyon 1, 8 avenue Rockefeller, 69373, Lyon cedex 08. davezies@univ-lyon1.fr. 